\title{
Magnetically driven transient phenomena in accretion driven systems: New breakthroughs with meerKAT and CTA?
}

\section{P.J. Meintjes ${ }^{* \dagger}$}

Department of Physics, University of the Free State, PO Box 339, Bloemfontein, South Africa

E-mail: MeintjPJ@ufs.ac.za

\begin{abstract}
The advent of an era with more sensitive telescopes across the electromagnetic spectrum, like e.g. meerKAT, Cherenkov Telescope Array (CTA) and the Large Synoptic Survey Telescope (LSST), will open-up new possibilities to study faint transient phenomena in the universe. In this study it is shown that the transient emission across the electromagnetic spectrum in nearly all classes of accretion driven systems can be linked to magnetohydrodynamic processes, like e.g. turbulence, magnetic reconnection, particle acceleration and non-thermal emission. The role of turbulence and magnetic reconnection is investigated in dwarf nova eruptions, especially the role it plays in the production of strong non-thermal synchrotron emission recently detected in these systems. The newly detected binary white dwarf pulsar AR Scorpii (AR Sco) is also investigated. It is shown that the strong magnetic interaction between a very highly magnetized white dwarf and magnetic M5 secondary star can explain the total emission in this system, from radio to possibly X-rays. The possibility is investigated whether AR Sco can be a Very High Energy (VHE) gamma-ray source, given its proximity of about $120 \mathrm{pc}$.
\end{abstract}

Accretion Processes in Cosmic Sources (APCS2016)

5-10 September 2016,

Saint Petersburg, Russia

\footnotetext{
* Speaker.

${ }^{\dagger}$ The author thanks the organisers for their invitation and hospitality.
} 


\section{Introduction}

The recent detection of non-thermal radio synchrotron emission at the $\mu \mathrm{Jy}$ flux level of several dwarf novae during outbursts ([1]) utilizing the Very Large Array (VLA), sparked renewed interest in the production processes of non-thermal emission in close accretion driven binaries during outburts. Multi-wavelength non-thermal emission from other cataclysmic variables, namely the propeller source AE Aquarii, have been reported, from radio to possibly VHE gamma-rays (e.g.[2, 3, 4, 5, 6]). Recently, another white dwarf binary system, namely AR Sco, has been detected showing non-thermal emission across the whole Spectral Energy Distribution (SED) from radio to possibly X-rays wavelengths ([7]). The common denominator in these reports of nonthermal emission from cataclysmic variables, is their proximity $(\sim 100-300 \mathrm{pc})$, and all are reasonably bright, which makes them the ideal laboratories to study multi-wavelength emission produced through a plethora of different processes.

The development of the high frequency component of the Square Kilometre Array (SKA) precursor, namely meerKAT in South Africa, plus the construction of the Large Synoptic Survey Telescope (LSST) in Chile and the development of the Northern and Southern nodes of the Cherenkov Telescope Array (CTA) provide unique opportunities for multi-wavelength astronomy in the near future. New landmarks in terms of sensitivity and resolution will be obtained from radio to VHE gamma-rays. This will definitely provide unique opportunities to study transient multi-wavelength emission in a variety of sources in unprecedented detail. In this paper the focus will be placed on the processes that could possibly drive non-thermal flaring in dwarf novae, as well as the multi-wavelength emission in the recently discovered binary white dwarf pulsar AR Sco ([7]).

The paper will be structured as follows: In the next section magnetohydrodynamic viscosity in turbulent accretion discs will be discussed, and how it relates to dwarf nova eruptions and nonthermal flares. In the following section the binary white dwarf pulsar AR Sco is discussed. A model is presented to unify the multi-frequency Spectral Energy Distribution (SED) from radio to optical frequencies. It will be shown that the reservoir that drives the multi-wavelength emission is the spin-down power from a rapidly rotating spun-up white dwarf. Conclusions are then presented.

\section{Magnetohydrodynamic Driven Accretion Discs: The Dwarf Nova Phenomenon and Non-Thermal Emission}

The quantification of accretion disc viscosity received enormous impetus with the incorporation of turbulent viscosity, which exceeds molecular viscosity by several orders of magnitude. In a seminal paper ([8]), the turbulent viscosity is parameterised by $v_{\text {turb }}=\alpha c_{\mathrm{s}} H$, where $\alpha<1$ is a dimensionless parameter which scales the magnitude of the viscosity in terms of the sound speed $c_{\mathrm{s}}$ and the disc scale height $H$. Following this model, the hysteresis S-curve characterizing a dwarf nova (DN) outburst in the $\Sigma-T_{\text {disc }}$ plane has been quantified in terms of a cyclic $\alpha$ parameter variation ([9]). Simulated DN lightcurves resemble observed lightcurves most closely for values of $\alpha_{\text {cold }} \sim 0.01$ and $\alpha_{\text {hot }} \sim 0.1-0.3$ ([10]). Although the introduction of turbulent viscosity through the $\alpha$ parameter proved to be a useful way of constraining the magnitude range of the disc viscosity, the absence of a detailed theory of turbulence means that it is no more than a neat way of 
hiding numerous ill-understood underlying principles. It has been shown that magnetized discs are unstable against the magnetorotational instability (MRI) ([11, 12, 13]). For example, [14] showed that the strong coupling between the disc plasma and weak disc magnetic fields via the Lorentz force results in the MRI being an effective mechanism for angular momentum transfer in accretion discs. This mechanism provides a more quantitative description of the viscosity variations between the "hot" and "cold" states of the accretion disc during the DN outbursts. In this study the MHD driven turbulence and viscosity will be investigated and an attempt will be made to quantify it in terms of disc magnetization and flow profiles, using the basic equations of magnetohydrodynamics in the near-ideal limit.

\subsection{Magnetic Transport and Viscosity}

The effect of magnetic viscosity will be investigated and expressed in terms of the magnetic transport through the disc, which is described effectively by two parameters, i.e. the magnetic Hartmann $(M)$ and Reynolds $\left(R_{\mathrm{m}}\right)$ numbers (e.g. [15]). The Hartmann number is the ratio of magnetic stresses to particle viscosity in a magnetized fluid, while the Reynolds number essentially represents the ratio of the convection and diffusion associated with the magnetic field in the fluid. These two numbers are given respectively by

$$
\begin{aligned}
M & =\left(\frac{\sigma B^{2} L^{2}}{\mu c^{2}}\right)^{1 / 2} \\
R_{\mathrm{m}} & =\left(\frac{4 \pi L v \sigma}{c^{2}}\right),
\end{aligned}
$$

where $\sigma, \mu(=\rho v), B, L$ and $v$ represent the fluid electrical conductivity, the coefficient of dynamical viscosity, the magnetic intensity, the length scale of the field and the bulk flow speed respectively. If both $M \geq 1$ and $R_{\mathrm{m}} \geq 1$ the magnetic field will play an extremely important role in the dynamics of a magnetized fluid, which can have important consequences in astrophysical environments like accretion discs.

\subsection{Magnetized Accretion Discs and Outbursts}

The accretion disc dynamics in compact objects containing a slow-rotator (e.g. [16]) is displayed in Figure 1. It can be seen that the azimuthal disc flow velocity is faster than the rotation velocity of the compact object inside the corotation radius, and slower outside.

The inner disc structure of magnetized accretion discs has been invesigated (e.g. [17, 18]). The radial inflow velocity $\left(\mathrm{v}_{\mathrm{R}}\right)$ of a magnetized accretion disc has been shown to scale like

$$
-\mathrm{v}_{\mathrm{R}} \propto\left(\frac{B_{*}^{2} R_{*}^{6}}{M_{*} \dot{M}^{2}}\right)^{13 / 19} \frac{1-\Omega_{*} / \Omega_{\mathrm{K}}}{\eta^{17 / 19} R^{108 / 19}},
$$

where $B_{*}, M_{*}, \dot{M}, \Omega_{*}=\left(\frac{2 \pi}{P_{*}}\right), \Omega_{\mathrm{K}}=\left(G M / r^{3}\right)^{1 / 2}$ and $\eta$ represent the polar value of the poloidal magnetic field, the mass of the compact object, the mass accretion rate, the spin angular velocity, the Keplerian angular velocity and the diffusivity of the plasma respectively. It can be seen that for slow rotators material inside the corotation radius satisfies $\Omega_{*} / \Omega_{\mathrm{K}}=P_{\mathrm{K}} / P_{*}<1$, resulting in an inward flow of material, while for regions in the accretion disc outside the corotation radius 


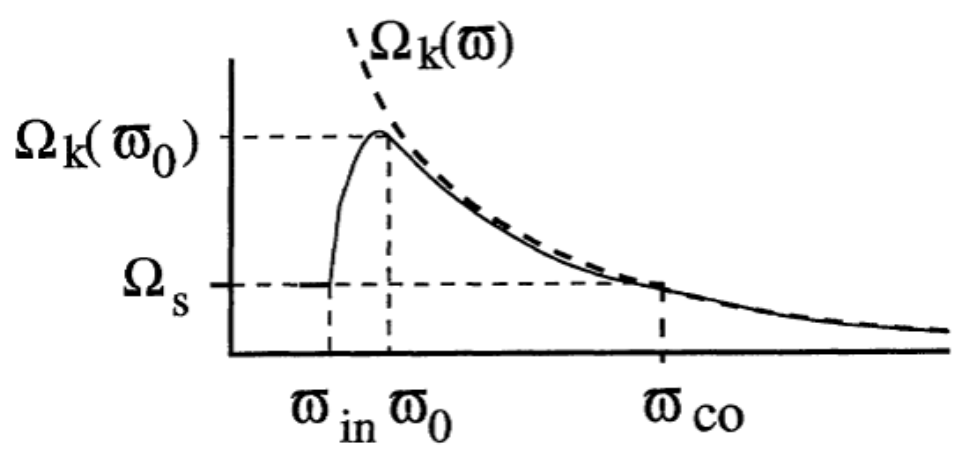

Disc angular velocity profile

Figure 1: Flow profile of a Keplerian accretion disc. Adopted from [16].

$\Omega_{*} / \Omega_{\mathrm{K}}=P_{\mathrm{K}} / P_{*}>1$, resulting in an outward magneto-centrifugal push ([16]) (see Figure 1) which effectively impedes the viscosity-driven radial inflow. The radial low profile in such a disc is illustrated in Figure 2 ([19]).

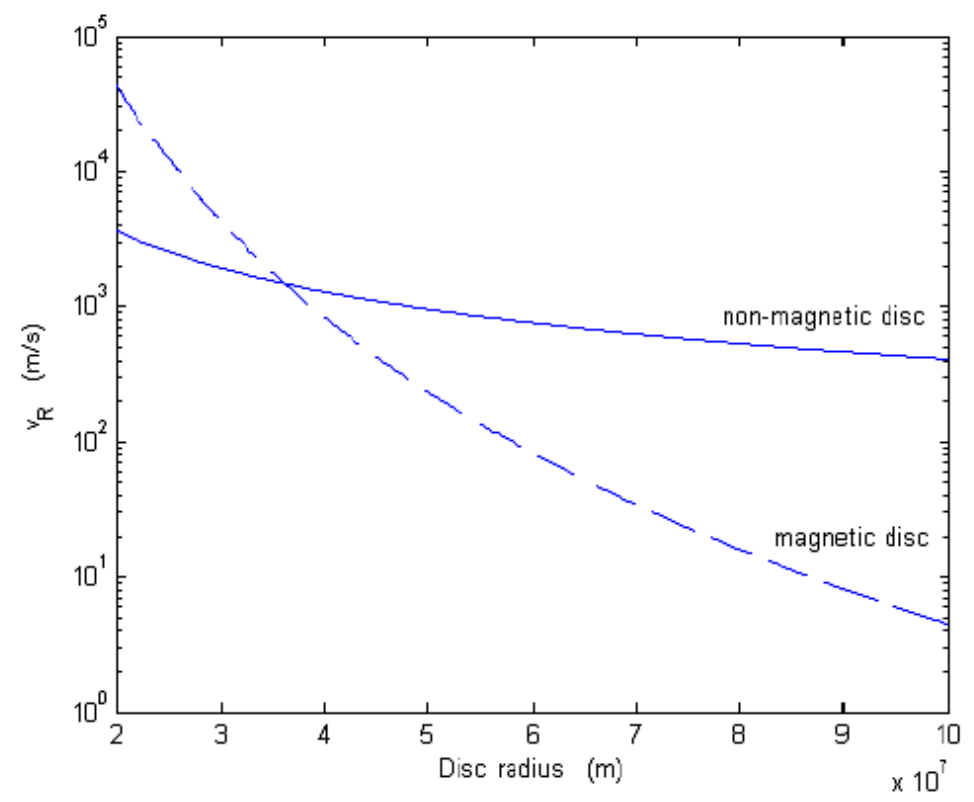

Figure 2: Radial inflow profile of a magnetized disc compared to a Keplerian accretion disc. Notable is the slower radial inflow speed outside the corotation radius in a magnetized disc. Adopted from [19].

Hydrodynamic stability in Keplerian accretion discs is preserved by the Rayleigh stability criterion (e.g. [20]), namely

$$
\frac{d\left(r^{2} \Omega\right)^{2}}{d r}>0
$$


However, it has been shown (e.g $[17,18,19])$ that flow-profiles in a magnetized disc deviate noticably from the flow in a Keplerion disc. Recent numerical simulations of accretion discs verify the presence of hydrodynamical turbulence (e.g. [21, 22]). These authors showed that a non-symmetric gravitational force causes a precessional wave in the disc, characterized by strong density and velocity gradients. This results in discs being subjected to small-scale radial instabilities with growth rate of about tenths to hundreths of the orbital period of the binary. They showed that if the variation of the velocity on length scales equal to the perturbation length scale is of the order of the sound speed or higher, these perturbations become unstable and the turbulence can grow on timescales equal to one period of the disc. The presence of turbulent plasma in the disc will have a significant effect on the disc properties like magnetic diffusivity and electrical conductivity, which quantifies the values of the magnetic Reynolds and Hartmann numbers, e.g. $R_{\mathrm{m}} \propto v \sigma$ and $M \propto\left(\sigma B^{2} / \rho v\right)^{1 / 2}$ (e.g. Equations. 2.1 and 2.2), where $v$ and $\sigma$ represent the kinematic viscosity and electrical conductivity respectively. It is proposed that the differential rotation between the compact object and disc will create a strong toroidal magnetic component (see e.g. Figure 3). This configuration will be condusive for the onset of the resistive tearing mode instability ([23]) and subsequent magnetic reconnection (e.g. [24] for a review), which will result in the disc being filled with magnetic islands, which will become buoyant due to the Parker buoyancy instability ([25]) resulting in the creation of a vertical magnetic field components (see Figure 4).

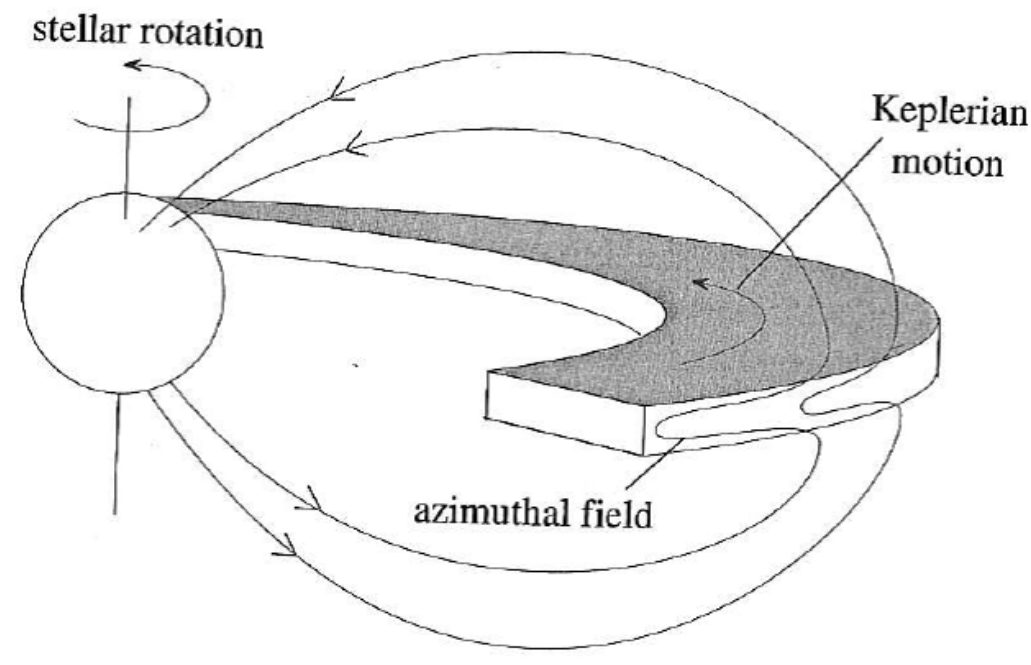

Figure 3: Strong toroidal field generation in magnetized accretion discs due to the differential rotation distorting the poloidal field of the accreting compact object. Adopted from [26].

\subsection{The Magneto-Gravitational Instability}

It is suggested that during the build-up phase towards an outburst the disc is growing outside the corotation radius, due to the slower inflow speed. This results in the accumulation of turbulent magnetized plasma. The Parker instability will result in the vertical field rising up and forming a magnetized carpet on both surfaces of the accretion disc (see e.g. [27]).

In the "cold" state the turbulent diffusivity of the disc plasma will result in the disc magnetic field to be lying mainly on the surface. However, these disc coronal fields will reconnect with the 
(a) Coroniti model

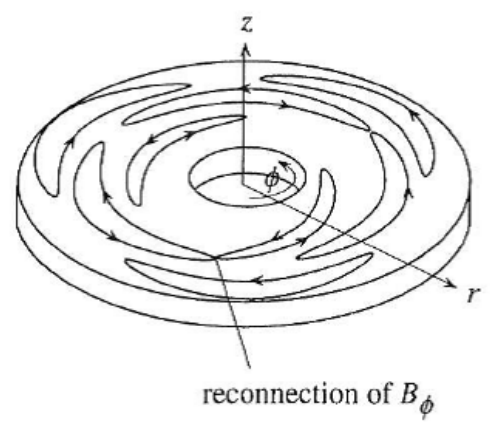

(b) Tout \& Pringle model

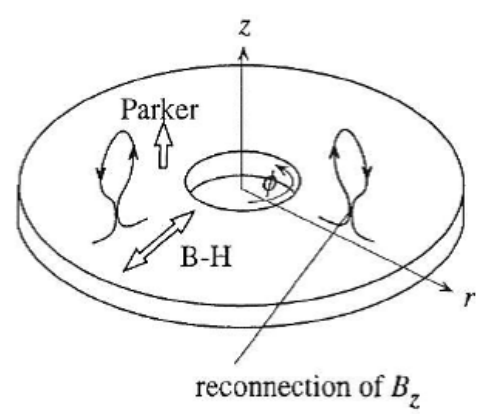

Figure 4: Turbulent magnetic field generation in an accretion disc. Strong toroidal fields will form (a) which will be subjected to the tearing mode and Parker instabilities, resulting in vertical magnetic fields (b) that drive the MRI instability as well as reconnection. This will drive disc heating and possible particle acceleration. Adopted from [26].

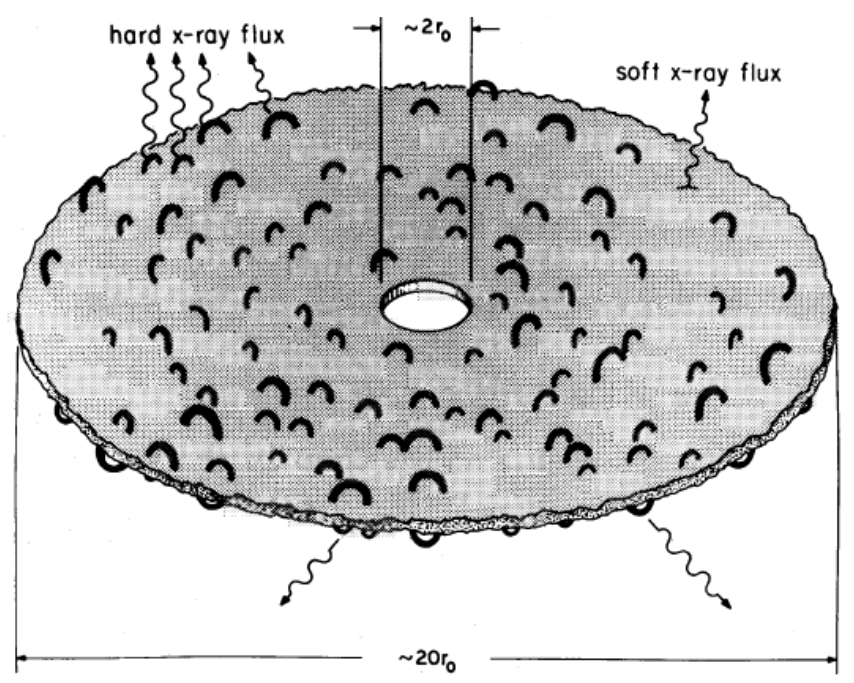

Figure 5: Turbulent magnetic field generation in an accretion disc producing a magnetized corona. Adopted from [27].

large scale poloidal fields of the compact object, resulting in plasma heating, and a subsequent increase in the disc's conductivity. It can be shown that if the disc conductivity has increased to values that results in the frozen-in condition to be largely retained, with disc fields below the surface reaching values of the order of $B_{\mathrm{d}} \geq 100$ Gauss, the magnetic Hartmann number may reach

$$
M \geq 1\left(\frac{B_{\text {disc }}}{100 \mathrm{G}}\right)\left(\frac{L}{H_{\text {disc }}}\right)\left(\frac{v_{\mathrm{T}}}{10^{13} \mathrm{~cm}^{2} \mathrm{~s}^{-1}}\right)^{-1 / 2}\left(\frac{\sigma_{\mathrm{T}}}{10^{7} \mathrm{~s}^{-1}}\right)^{1 / 2}
$$

with the kinematic viscosity $v_{\mathrm{T}}=\alpha H_{\mathrm{disc}} c_{s} \sim 10^{13}(\alpha / 0.1)\left(H_{\mathrm{disc}} / 10^{8} \mathrm{~cm}\right)\left(c_{\mathrm{s}} / 10^{6} \mathrm{~cm} \mathrm{~s}^{-1}\right) \mathrm{cm}^{2} \mathrm{~s}^{-1}$ and the magnetic diffusivity $\eta_{\mathrm{T}} \sim\left(c^{2} / 4 \pi \sigma_{\mathrm{T}}\right) \sim 10^{13} \mathrm{~cm}^{2} \mathrm{~s}^{-1}$, which is condusive for the trigger of 
a Magneto-Gravitational Instability ([28, 29, 30]). This implies that in the frame of the fluid, the "frozen-in" magnetic content of the disc inhibits slippage of plasma through, or across, the disc fields, hence decelerating it. This can be quantified by the effective gravity perpendicular to the rotating disc flow, namely

$$
\mathbf{g}_{\mathrm{eff}, \perp}=\left(-\frac{G M_{1}}{R^{2}}+\frac{\mathrm{v}_{\phi}^{2}}{R}\right) \hat{\mathbf{e}}_{\mathrm{R}},
$$

with $R$ being the distance from the center of the compact accreting object in the equatorial plane of the binary. From these equations $\mathbf{g}_{\mathrm{eff}, \perp} \rightarrow 0$ for Keplerian flow, i.e. $\mathrm{v}_{\phi} \rightarrow \mathrm{v}_{\mathrm{K}}$. However, one can see that if the azimuthal flow is decelerated across the fields, $\mathbf{g}_{\mathrm{eff}, \perp}<0\left(\mathrm{v}_{\phi}<\mathrm{v}_{\mathrm{K}}\right)$. This means that if the disc has reached critical levels of magnetization, for which the Hartmann number $M \geq 1$, the azimuthal flow will be decelerated such that the effective gravity will point radially inwards, which implies that during these states the disc plasma will experience a net inward pull towards the compact accreting object, leading to a large scale inward advection of disc plasma towards the compact object, draining the bulk of the disc onto the compact object. The inward advection will result in the magnetic field anchored in the disc, to be advected with the inward flow (see Figure 6). It is believed that this process provides a suitable platform to explain the recent detections of non-thermal radio synchrotron flares from a few dwarf novae during outbursts (see [1]).
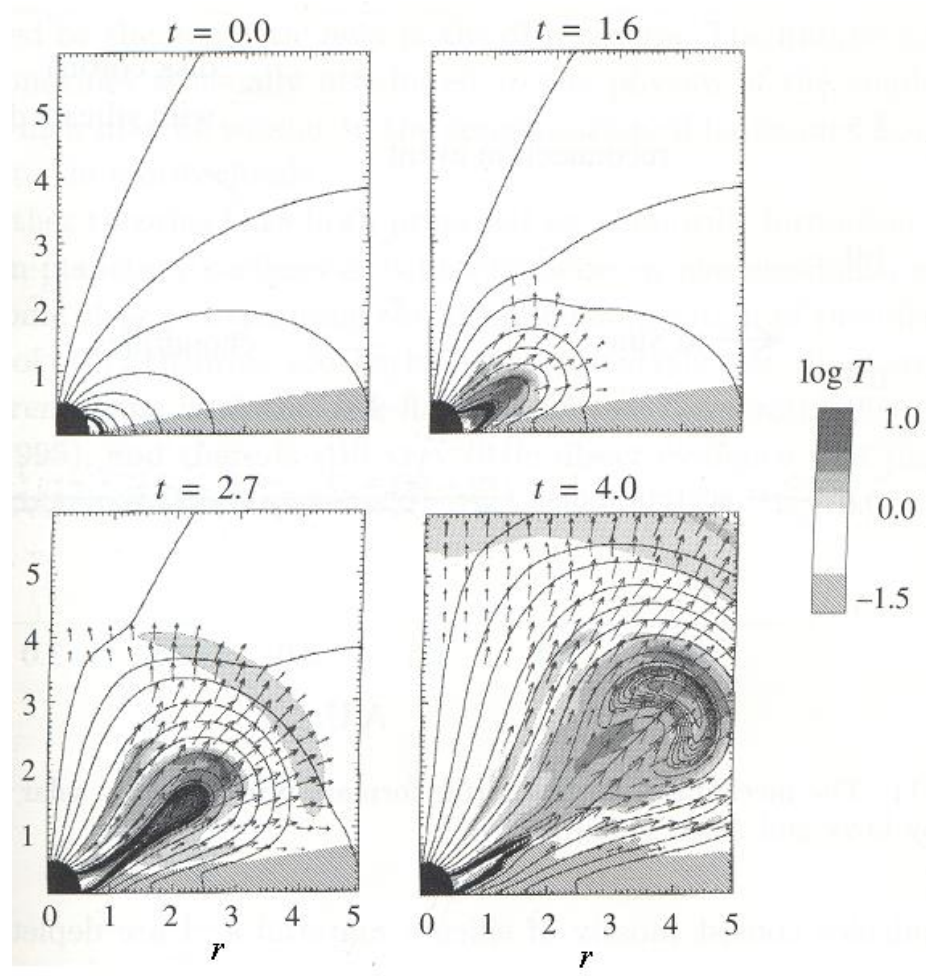

Figure 6: Magnetic advection during dwarf nova erruptions, leading to the formation of magnetic bubbles and subsequent reconnection. Adopted from [31, 26]. 


\subsection{Dwarf Novae: Non-Thermal Outbursts and meerKAT Follow-up}

Recent VLA observations of a sample of novalike variables ([1]) revealed detectable nonthermal synchrotron emission from a sample of dwarf novae sources. The distance to these sources ranges between 100-335 pc, with peak emission ranging between 10-240 $\mu \mathrm{Jy}$ ([1]). Spectral information of two sources, namely V603 Aql and TT Ari, reveal a $S_{v} \propto v^{\alpha}$ spectrum, typically associated with expanding synchrotron emitting clouds which cool as they expand, i.e. the socalled van der Laan process ([32]). A superposition of these flares will produce a typical $S_{v} \propto v^{\alpha}$ spectrum (e.g. [3]), with $\alpha<1$.

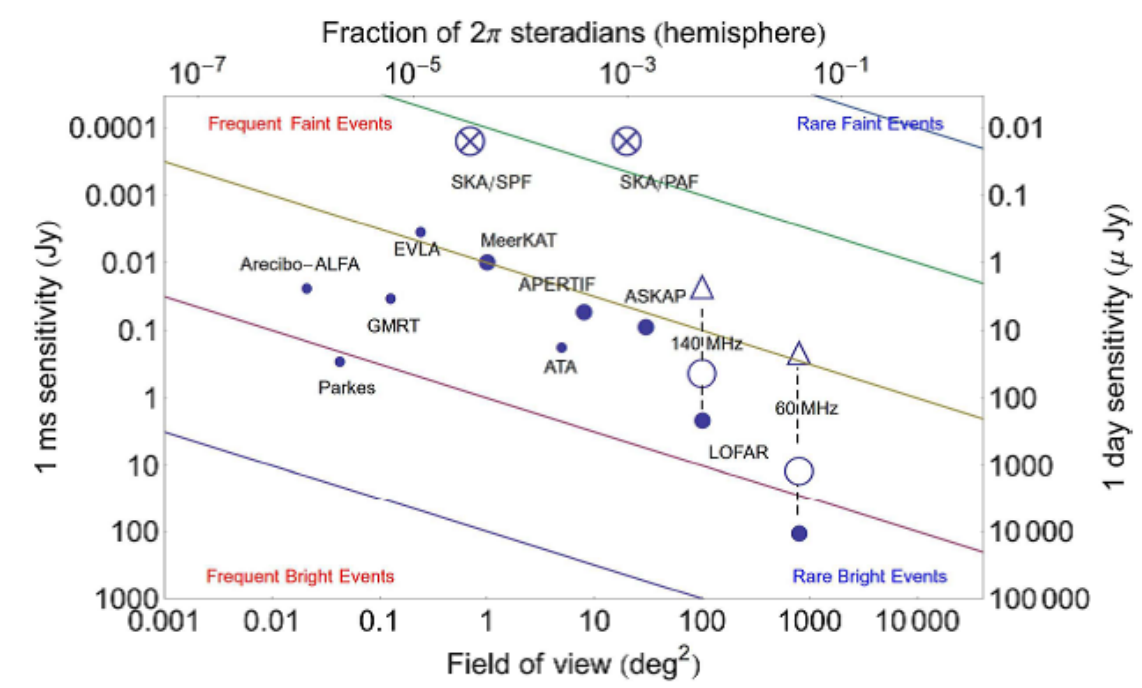

Figure 7: Current radio telescope facilties sensitivity levels. Adopted from [33].

The radio flux estimates of dwarf nova outbursts have recently been investigated ([34]) utilizing the van der Laan model. It has been shown that the initial flux of an expanding synchrotron bubble associated with a source at a distance of approximately $100 \mathrm{pc}$, is approximately

$$
\begin{aligned}
S_{\mathrm{m}, \circ} \approx & 60\left[\left(\frac{\varepsilon_{\mathrm{e}}}{1 \mathrm{MeV}}\right)^{1.75}\left(\frac{r_{\circ}}{10^{9} \mathrm{~cm}}\right)^{6.67}\left(\frac{N_{\circ}}{10^{9} \mathrm{~cm}}\right)\left(\frac{B_{\circ}}{100 \mathrm{Gauss}}\right)^{0.958}\right]^{0.3529} \times \\
& \left(\frac{v_{\mathrm{m} \circ}}{60 \mathrm{GHz}}\right)^{1.3088} \mu \mathrm{Jy},
\end{aligned}
$$

for an electron power-law spectrum with index $\delta=2.75$. The frequency where the initial flux peaks is then

$$
\nu_{\circ}=60\left(\frac{\varepsilon_{\mathrm{e}}}{\mathrm{MeV}}\right)^{0.5185}\left(\frac{N_{\circ}}{10^{9} \mathrm{~cm}^{-3}}\right)^{0.2963}\left(\frac{r_{\circ}}{10^{9} \mathrm{~cm}}\right)^{0.2963}\left(\frac{B_{\circ}}{100 \mathrm{G}}\right)^{0.703} \mathrm{GHz} .
$$

This estimate provides interesting possibilities for future studies with meerKAT and the SKA. At frequncies below $v_{\circ}$ the flux spectrum will typically follow a self-absorbed $S_{v} \propto v^{\alpha}$ spectrum (e.g. [3]). 
The expected meerKAT and SKA sensitivities have recently ([33]) been put on display with other radio telescope facilities. It can be seen that meerKAT and the VLA will both be on the $~$ $100 \mu \mathrm{Jy}$ (1 day) sensitivity level, with the advantage that meerKAT will have a 10 times larger field of view (FoV) than the VLA, making it a much improved survey instrument. However, the SKA will significantly improve on this, reaching $\sim 1 \mu \mathrm{Jy}$ ( 1 day) sensitivity levels with a larger FoV, making it the near-perfect survey instrument to search for radio transients which can be associated with dwarf novae and other accretion driven transients.

\section{AR Sco: A Spin-Powered Binary White Dwarf Pulsar}

The discovery of a binary spin-powered white dwarf pulsar AR Sco has been announced ([7]). This system shows multi-wavelength emission from radio to possibly X-rays, with strong pulsations at the white dwarf spin period $\left(P_{\text {spin }}=117 \mathrm{~s}\right)$ and a beat period $P_{\text {beat }}=118 \mathrm{~s}$ with the binary orbit $\left(P_{\text {orb }}=3.56 \mathrm{~h}\right)$. The pulsed signal strength is remarkable, significantly higher than any other accretion driven magnetic cataclysmic variable ([7]). The multi-wavelength Spectral Energy Distribution (SED) is shown in Figure 8.

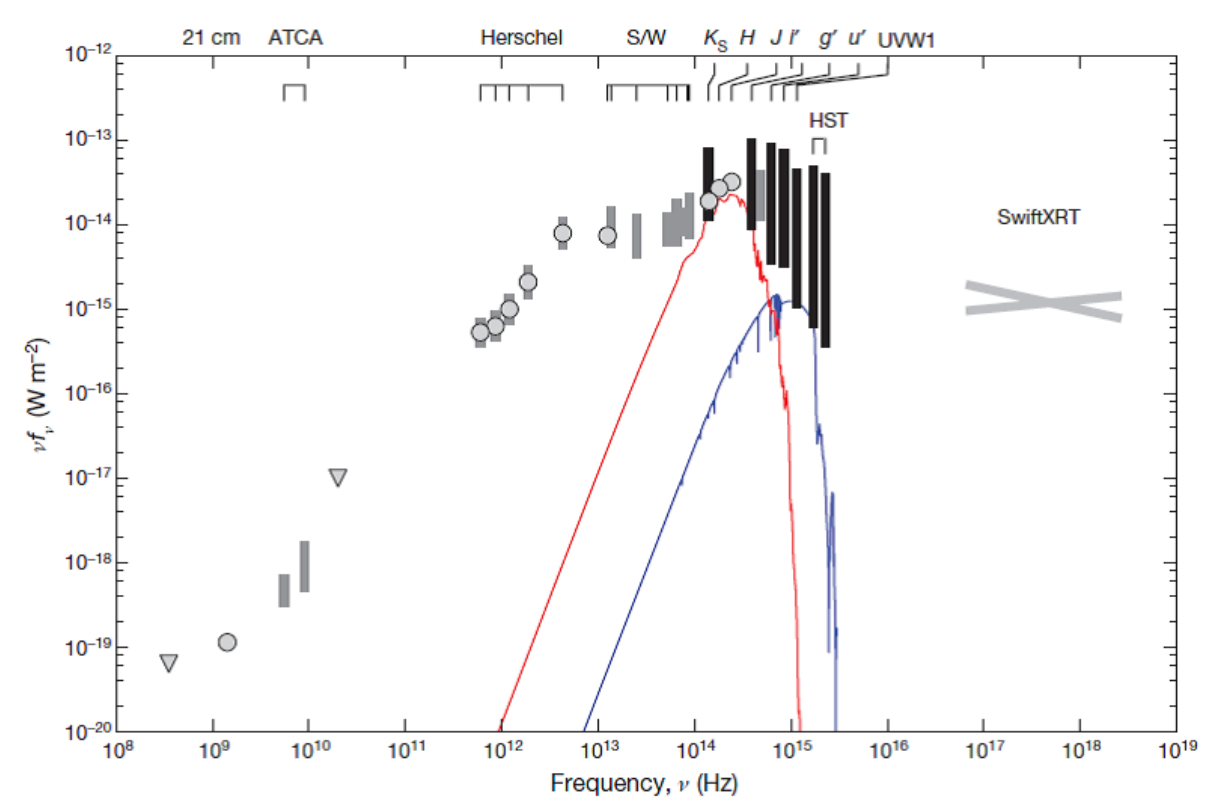

Figure 8: Multiwavelength SED of AR Sco. Adopted from [7].

It can be seen that the white dwarfs in both AR Sco and AE Aqr behave like spin-powered pulsars (see. [36]). In a recent study ([35]) an emphatic model has been proposed that unites the total SED of AR Sco from radio to X-ray wavelengths. These authors proposed that the total pulsed component of the SED, from radio to optical, may be the result of non-thermal synchrotron emission produced by a pulsar-mechanism in the magnetosphere of the fast rotating white dwarf which pumps the coronal loops of the secondary star. They proposed that the SED below frequencies $v \sim$ few $\times 10^{12} \mathrm{~Hz}$ and above frequencies $v \sim 10^{13} \mathrm{~Hz}$ are indeed separate spectra, with the lower frequency component possibly produced by synchrotron radiation in the pumped coronal loops of 


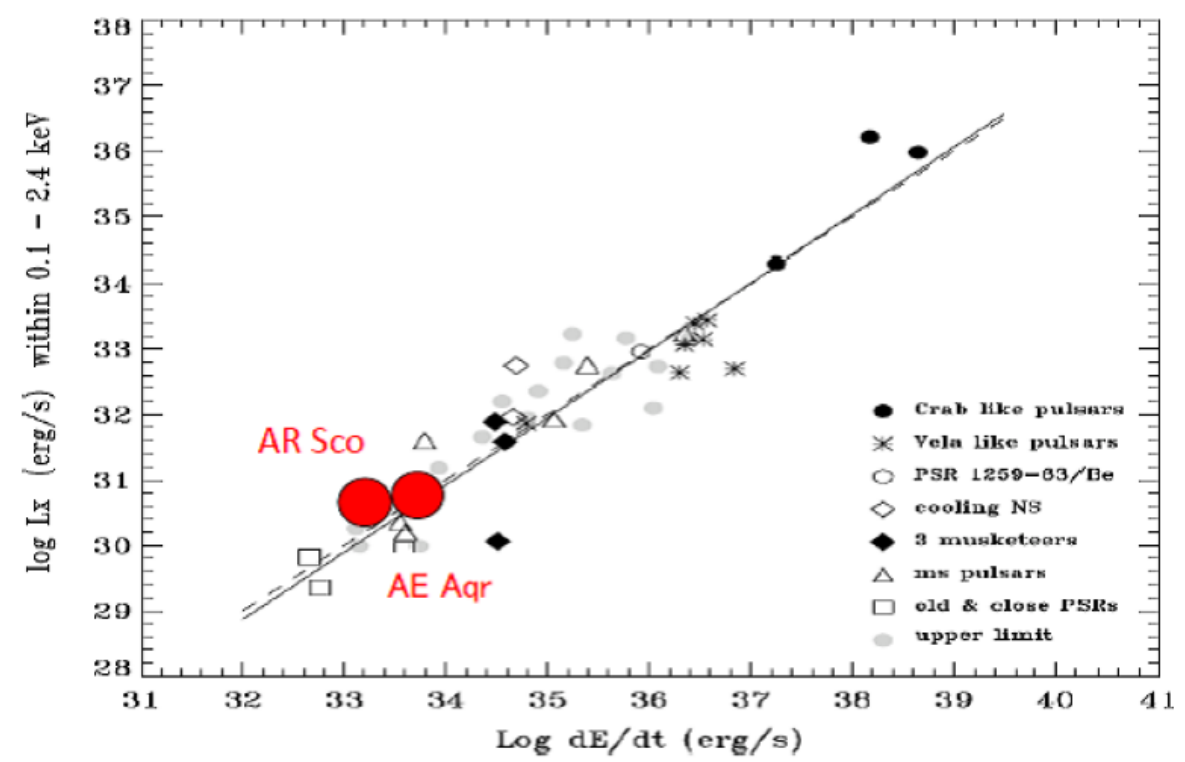

Figure 9: X-ray luminosity vs spin-down power of the white dwarf in AR Sco and AE Aquarii. Adopted from [35], which adapted it from [36].

the $\sim 0.3 M_{\odot}$ M5 secondary star through a van der Laan process $\left(S_{v} \propto v^{\alpha_{1}}\right)$, with the higher frequency spectrum with a negative slope, i.e. $S_{v} \propto v^{-\alpha_{2}}$, being the result of optically thin synchrotron emission in the striped pulsar wind (e.g. [37]) of the highly magnetized white dwarf.

\subsection{The Model: An Executive Summary}

It has been mentioned that the multi-wavelength SED of AR Sco shows a distinct non-thermal nature, from radio to optical (and perhaps also X-rays), and since the cadence across the spectrum is sufficient, it can be seen that the SED presented by ([7]), converted in this study to differential flux $\left(S_{v}\right)$, can be divided into two parts, i.e. $v \leq$ few $\times 10^{12} \mathrm{~Hz}$, with $S_{v} \propto v^{\alpha_{1}}$, with $\alpha_{1} \sim 0.4$, and an optically thin synchrotron component, with $S_{v} \propto v^{-\alpha_{2}}$, with $\alpha_{2} \sim 1.4$. This spectral index is very similar to the photon index inferred from the hard X-ray spectrum deduced form the Suzaku data of the other binary spin-powered pulsar, namely the white dwarf in AE Aquarii (which is $\alpha=1.2$ ) ([40]), as well as other spin-powerd pulsars. Since $\alpha_{2}=(p-1) / 2$, with $p$ being the power-law index of the relativistic electrons, it implies that for AR Sco and AE Aqr the power law electron spectral indexes are $p=3.8$ (AR Sco) and $p=3.4$ (AE Aquarii) respectively. If the total reservoir of the spin-down power of the white dwarf, i.e. $\dot{E}_{\mathrm{S}-\mathrm{d}} \sim 10^{33} \mathrm{erg} \mathrm{s}^{-1}$, is radiated in magnetic dipole radiation, a constraint can be placed on the white dwarf magnetic field, which is of the order of $B_{*} \leq 500 \mathrm{MG}$, which is high for a binary white dwarf, although there are isolated white dwarfs with higher fields ([7]).

It has been suggested ([35]) that the nature of the periodicity, namely the presence of the white dwarf spin and orbital beat periods can be reconciled with the fact that the whole binary, i.e. $a \sim 8 \times 10^{10}\left(M_{\mathrm{wd}} / 0.8 M_{\odot}\right)^{1 / 3}\left(P_{\mathrm{orb}} / 3.5 \mathrm{hr}\right)^{2 / 3} \mathrm{~cm}$ fits inside the light cylinder radius (accelerator zone) of the white dwarf pulsar, which is $r_{1-\mathrm{c}} \sim 6 \times 10^{11}\left(\Omega_{w d} / 0.054 \mathrm{rad} \mathrm{s}^{-1}\right) \mathrm{cm}$. Therefore, it is 
anticipated that the periodic emission produced by synchrotron radiation in the relativistic pulsar wind will have a signature of both the spin and the beat periods. A characteristic of the spectrum below $v \sim$ few $\times 10^{12} \mathrm{~Hz}$ is the presence of pulsed emission, predominantly at the beat period. It has been shown ([35]) that this can be attributed to synchrotron emission of mildly relativistic electrons trapped in the pumped coronal fields of the secondary star. It has been shown that the MHD power dissipated into the surface layers of the secondary star, as a result of the pumping effect of the white dwarf, is of the order of $P_{\text {mhd }} \sim$ few $\times 10^{31} \mathrm{erg} \mathrm{s}^{-1}$, which can comfortably drive the line emission, which is confined to the secondary star's surface facing the white dwarf ([7]). It can also be shown that this dissipation of MHD power in the envelope of the M5 dwarf, can tidally lock the two stars over a period of approximately $\tau \sim 10^{7} \mathrm{yr}$, which is similar to the inferred spin-down timescale of the white dwarf ([7]).

\subsection{Possible VHE Emission from AR Sco}

It has been shown that the ratio of the X-ray luminosity $\left(L_{\mathrm{X}} \approx 10^{30} \mathrm{erg} \mathrm{s}^{-1}\right)$ to spin-down luminosity $\left(L_{\mathrm{s}-\mathrm{d}}=I \Omega \dot{\Omega} \approx 10^{33} \mathrm{erg} \mathrm{s}^{-1}\right)$, namely $\alpha \sim\left(L_{\mathrm{x}} / L_{\mathrm{s}-\mathrm{d}}\right) \sim 10^{-3}$ ([35, 36]), implies that the bulk of the emission from the system is not produced by the accretion of matter and that the white dwarf is acting like a spin-powered pulsar. Potentials of the order (e.g. [38, 39, 40])

$$
\Delta V \sim 10^{12}\left(\frac{P_{*}}{117 \mathrm{~s}}\right)^{-5 / 2}\left(\frac{\mu}{8 \times 10^{34} \mathrm{G} \mathrm{cm}^{3}}\right)\left(\frac{R_{*}}{5.5 \times 10^{8} \mathrm{~cm}}\right) \text { Volt }
$$

can be induced between the white dwarf and the light cylinder. Since this electric field is several orders of magnitude stronger than the surface gravity on the white dwarf, charged particles like electrons can be pulled from the crystal lattice of these degenerate stars, and accelerated to relativistic energies of the order of $\gamma_{\mathrm{e}} \leq 10^{6}$, resulting in single-electron synchrotron emission up to to frequencies of the order of

$$
v_{\mathrm{syn}} \leq 10^{18}\left(\frac{B_{1-\mathrm{c}}}{0.4 \mathrm{G}}\right)\left(\frac{\gamma_{\mathrm{e}}}{10^{6}}\right)^{2} \mathrm{~Hz}
$$

with peak emission occurring $v_{\mathrm{c}} \sim 0.3 v_{\mathrm{syn}} \leq 3 \times 10^{17} \mathrm{~Hz}$.

The maximum energy transfer for potential inverse Compton processes as a result of $\gamma$-ray interactions within the $\rho$ Ophiuchus cloud complex can be calculated in the Thomson limit as

$$
\begin{aligned}
\varepsilon_{\gamma} & =4 \gamma^{2} \varepsilon_{\mathrm{ph}} \\
& \leq 4\left(\frac{\gamma}{10^{6}}\right)^{2}\left(\frac{\varepsilon_{\mathrm{ph}}}{0.1 \mathrm{eV}}\right) \mathrm{TeV}
\end{aligned}
$$

where $\gamma$ represents the electron Lorentz factor and where $\varepsilon_{\text {ph }}$ represents the background photon energy, presumably from the molecular cloud complex.

The possibility of a powerful particle accelerator being present in the AR Sco system opens-up interesting possibilities for future studies with powerful and sensitive new gamma-ray facilities like the Cherenkov Telescope Array (CTA), which provides more than a factor of 10 improvement in sensitivity over the current largest Cherenkov facilities, like the High Energy Sterioscopic System (H.E.S.S.) in Namibia (see e.g [41] for a review). 


\section{Conclusions}

In this study a few examples have been highlighted of the potential for non-thermal emission in close binary systems due to magnetohydrodynamic action in accretion discs, or in the magnetohydrodynamic action between two magnetic stars. It is shown that future telescopes, like for example meerKAT and the SKA, as well as the future Cherenkov Telescope Array (CTA) will be "game changers" in the field of multi-wavelength astronomy. Also, it is anticipated that the Large Synoptic Survey Telescope (LSST) will discover thousands of new variable stars, which will really provide endless possibilities for follow-up studies and the discovery of new surprises.

\section{References}

[1] D.L. Coppejans, E.G. Körding, J.C.A. Miller-Jones, M.P. Rupen, C. Knigge, G.R. Sivakovv \& P.J. Groot, Novalike cataclysmic variables are significant radio emitters, MNRAS 4513801 3813 (2015)

[2] J.A. Bookbinder \& D.Q. Lamb, Discovery of radio emission fro AE Aquarii, ApJ 323 L131 (1987)

[3] T.S. Bastian, G.A. Dulk \& G. Chanmugam, Radio Flares from AE Aquarii: A Low power Analogue to Cyg X-3, ApJ 324431 (1988)

[4] C.C.G. Bowden et al., 350 GeV gamma-rays from AE Aquarii, Astropart. Phys. 147 (1992)

[5] P.J. Meintjes et al., AE Aquarii: An emitter of pulsed TeV gamma-rays resembling optical emission during flares, ApJ 401325 (1992)

[6] P.J. Meintjes et al., Simultaneous optical and TeV gamma-ray observations of the cataclysmic variable AE Aquarii, ApJ 434292 (1994)

[7] T.R. Marsh et al., A radio pulsing white dwarf binary star, Nature, (doi:10.1038/nature18620) 1 (2016)

[8] N.U. Shakura \& R.A. Sunyaev, Black holes in binary systems. Observational appearance, A\&A 24337 (1997)

[9] F. Meyer \& E. Meyer-Hofmeister, On the elusive cause of cataclysmic variable outbursts, $A \& A 104$ L10 (1981)

[10] A Ichikawa \& Y. Osaki, Time evolution of the accretion disk radius of a dwarf nova, PASJ 44 $15(1992)$

[11] E.P Velikhov, Stability of an ideally conducting fluid between cylinders rotating in a magnetic field, Sov. Phys. JETP 361389 (1959)

[12] S. Chandrasekhar, The stability on non-dissipative Couette flow in hydromagnetics, Proc. Nat. Acad. Sci 46 253-257 (1960) 
[13] S. Chandrasekhar, Hydrodynamic and hydromagnetic stability, (Dover Publications Inc, New York) pp. 382-427 (1961)

[14] S.A. Balbus \& J.F. Hawley, A powerful local shear instability in weakly magnetized disks I Linear analysis. II - Nonlinear evolution, ApJ 376214 (1991)

[15] J.D. Jackson, Classical Electrodynamics, 2 nd ed., Wiley and Sons, New York, pp. 473, 477479 (1975)

[16] J. Li \& D.T Wickramasinghe, On the fastness parameter and the inner disc radius, MNRAS 286 L25 (1997)

[17] C.G. Campbell, The interaction of a magnetic neutron star with an accretion disc, MNRAS 229405 (1997a)

[18] C.G. Campbell, Magnetohydrodynamics in Binary Stars, Kluwer Academic Publishers, Dordrecht/Boston/London, pp. 171-210 (1997b)

[19] E. Breedt, A magnetohydrodynamic perspective on magnetic viscosity and mass transfer in disc accreting cataclysmic variables, M.Sc Thesis (Unpublished), University of the Free State (2005)

[20] D. Biskamp, Magnetohydrodynamic Turbulence, Cambridge university Press, Cambridge, p. $51(2003)$

[21] D.V. Bisikalo \& A.G. Zhilkin, Accretion processes in intermediate polars with asynchronous rotations of white dwarfs, in Proceedings of Golden Age of Cataclysmic Variables and Related Objects, Palermo, (eds. F. Giovanelli \& L. Sabau-Graziati), MEMORIE S.A.It 83 (2) 562 (2012)

[22] D. Bisikalo, E. Kurbatov \& P. Kaigorodov, On a possible mechanism of developing of the turbulence in accretion disks in nonmagnetic binary stars, in Proceedings of the MultiFrequency Behaviour of High Energy Cosmic Sources Workshop (MULTIF15), 25-30 May 2015, Palermo (Italy), ed. F Giovannelli, Proceedings of Science (PoS), Bisikalo-2015MULTIF15-043 (2015)

[23] H.P. Furth, J. Killeen \& M.N. Rosenbluth, Finite-resistivity instabilities of a sheet pinch, Phys. Fluids 16 459-484 (1963)

[24] E.G. Zweibel \& M. Yamada, Magnetic reconnection in astrophysical and laboratory plasmas, Annu. Rev. Astron. Astrophys. 47 291-332 (2009)

[25] E.N. Parker, The dynamical state of the interstellar gas and field, ApJ, 145811 (1966)

[26] E. Priest \& T. Forbes, Magnetic Reconnection: MHD theory and application, Cambridge Univ. Press, Cambridge, pp. 441-457 (2000)

[27] A.A. Galeev, R. Rosner \& G.S. Vaiana, Structured Coronae of Accretion Disks, ApJ 229318 (1979) 
[28] P.J. Meintjes \& E. Breedt, Magnetic viscocity: Outbursts and outflows in accretion driven systems, in Proceedings of High Energy Astrophysics in Southern Africa: A multi-frequency perspective of new frontiers in high energy astrophysics in Southern Africa (eds. B van Soelen \& P. Meintjes) Mem. S.A. It Vol 86 (1) 89 (2015)

[29] P.J. Meintjes, E. Breedt \& M. Erwee, Magnetized Accretion Discs and the Dwarf Nova Phenomenon, in Proceedings of Frontier Research in Astrophysics (eds. F. Giovanelli, J. Beall, S. Colafeancesco, D. Bisikalo and L. Sabau-Graziati) Proceedings of Science PoS(FRAPWS2014)010 (2015)

[30] P.J. Meintjes, Transient sources in astrophysics: From radio to Gamma-Rays, in proceedings of 3rd Annual Conference on High Energy Astrophysics in Southern Africa (ed. S. Razzaque), Proceedings of Science (2015) PoS(HEASA2015)004 (2015)

[31] M.R. Hayashi, K. Shibata \& R. Matsumoto, X-ray flares and mass outflows driven by magnetic interaction between a protostar and its surrounding disc, ApJ 468 L37 (1996)

[32] H. van der Laan A model for variable extragalactic radio sources, Nature 2111131 (1966)

[33] R. Fender, The scientific potential of LOFAR for time domain astronomy, in Proceedings of New Horizons and Time Domain Astronomy: IAU Symposium 285 (2011) eds. E. Griffen, R Hanisch \& R Seaman, arXiv:1112.2580V1 (astro-ph.HE) 12 December 2011

[34] P.J. Meintjes, Magnetic Reconnection and Transient Phenomena in Accretion Driven Systems in Proceedings of the 4th High Energy Astrophysics in Southern Africa Workshop (HEASA2016), 25-27 August 2016, Cape Town, South Africa, ed. M Bottcher (2016)

[35] D.A.H. Buckley, P.J. Meintjes, S.B. Potter, T.R. Marsh, B.T. Gänsicke, Polarimetric evidence of a white dwarf pulsar in the binary system AR Scorpii, Nature Astronomy 1 (2017) 0029

[36] W. Becker \& J. Trümper, The x-ray luminosity of rotation powered neutron stars, A\&A 326 682 (1997)

[37] J.G. Kirk, Y. Lyubarsky J. Pétri, The theory of pulsar winds and nebulae, Astrophysics and Space Science Library: Neutron Stars and Pulsars ed. W. Becker, 357 pp. 421-450 (2009)

[38] J. Arons \& E.T. Scharlemann, Pair formation above polar caps-structure of the low altitude accelerator zone, ApJ 231854 (1979)

[39] N Ikhsanov, The pulsar-like white dwarf in AE Aquarii, A\&A 338521 (1998)

[40] B. Oruru \& P.J. Meintjes, X-ray characteristics and spectral energy distribution of $A E$ Aquarii, MNRAS 4211557 (2012)

[41] B.S. Acharya, Introducing the CTA concept, Astropart. Phys. 433 (2012) 